\title{
Influence of racemic higenamine on the sinus node
}

\author{
FENGXIA YU ${ }^{1}$, LINGTING KONG ${ }^{1}$ and SHUJUAN WANG ${ }^{2}$ \\ ${ }^{1}$ Department of Emergency Medicine and ${ }^{2}$ Electrocardiogram room, Yantaishan Hospital, Yantai, Shandong 264001, P.R. China
}

Received September 5, 2012; Accepted October 25, 2012

DOI: $10.3892 /$ etm.2012.813

\begin{abstract}
The aim of this study was to explore the mechanism of racemic higenamine in the treatment of sick sinus syndrome (SSS). A total of 40 New Zealand rabbits were randomly divided into normal sinus node and damaged sinus node (SND) groups, and each group was randomly divided into treatment and control groups $(n=10)$. The SND model was established by formaldehyde wet dressing of the sinus node area. The treatment groups were administered an intravenous infusion of $0.04 \mathrm{mg} /$ $\mathrm{kg}$ racemic higenamine via the marginal ear vein within $5 \mathrm{~min}$. The electrophysiological indicators of sinoatrial function, including the sinus node recovery time (SNRT), corrected sinus node recovery time (CSNRT), total sinoatrial conduction time (TSACT) and sinus cycle length (SCL), were determined before and $20 \mathrm{~min}$ after medication and the changes in these indicators were evaluated. The two control groups were administered $10 \mathrm{ml}$ physiological saline. Following the administration of racemic higenamine, the SNRT, CSNRT, TSACT and SCL in the normal sinus node and SND groups were significantly shortened compared with those in the control groups $(\mathrm{P}<0.01)$. The electrophysiological influence of racemic higenamine on sinoatrial function in the SND group was significantly greater than that in the normal sinus node group $(\mathrm{P}<0.01)$, and its effect in the treatment of arrhythmia caused by a damaged sinus node was statistically significant $(\mathrm{P}<0.05)$. The main electrophysiological mechanism of racemic higenamine in the treatment of SSS was the enhancement of sinus node self-discipline and improvement of sinoatrial and atrioventricular conduction function.
\end{abstract}

\section{Introduction}

Sick sinus syndrome (SSS) is a common disease, which is mainly caused by sinus node dysfunction. Presently, western medicine has a poor curative effect on SSS. The implantation of a permanent cardiac pacemaker may effectively relieve the clinical symptoms, but requires complex technolo-

Correspondence to: Dr Fengxia Yu, Department of Emergency Medicine, Yantaishan Hospital, No. 91 Jiefang Road, Yantai, Shandong 264001, P.R. China

E-mail: fengxiayucn@163.com

Key words: sick sinus syndrome, racemic higenamine, sinus node recovery time, sinoatrial conduction time gies, expensive costs and invasive surgery, which limits the clinical applications. Therefore, pharmacotherapy remains an important measure (1). Traditional Chinese medicine has investigated SSS from the principle of dialectical differentiation, which has numerous advantages compared with western medicine. Aconite was a commonly used traditional Chinese medicine for the treatment for SSS, and racemic higenamine has been separated from it as the effective component mixed with crudes (2). Racemic higenamine may diminish inflammation, improve blood supply to the sinus node and enhance the ventricular rate (3-5). To date, there are no studies focused on the effect of racemic higenamine on damaged sinoatrial function. In the present study, an in vivo damaged sinus node (SND) model was established using live animals and a direct sinoatrial electrode pacing, in order to investigate the pathogenesis of SSS, and observe the electrophysiological influence of racemic higenamine on normal and damaged sinoatrial function in rabbits, and provide an experimental basis for the traditional Chinese medicinal treatment of SSS in the clinic.

\section{Materials and methods}

Animal grouping. A total of 40 New Zealand rabbits were randomly divided into two groups: normal sinus node and SND groups, with 20 rabbits in each group. In the normal sinus node group (sham-operated group), a physiological saline wet dressing was applied to each of the 20 rabbits. In the SND group, 20 rabbits were used to establish damaged sinus node models by the application of a formaldehyde wet dressing. The two groups were each randomly divided into treatment and control groups. The two treatment groups were treated with $0.04 \mathrm{mg} / \mathrm{kg}$ racemic higenamine via the marginal ear vein within $5 \mathrm{~min}$ and after $20 \mathrm{~min}$, the electrophysiological indicators of sinoatrial function were determined. These indicators included sinus node recovery time (SNRT), corrected sinus node recovery time (CSNRT), total sinoatrial conduction time (TSACT) and sinus cycle length (SCL). The two control groups were administered $10 \mathrm{ml}$ physiological saline.

Electrocardiogram recording. The electrocardiogram limb wires were inserted under the skin of limbs with electrodes (pin), and fixed with tape. Ten cardiac cycle electrocardiograms were recorded with a paper speed of $50 \mathrm{~mm} / \mathrm{sec}$.

Establishment of the SND model. A wire twined with cotton dipped into $36 \%$ formaldehyde was placed in the border zone 
Table I. Electrophysiological comparison between normal sinus node and damaged sinus node (SND) groups (mean \pm SD).

\begin{tabular}{lcccc}
\hline Groups & SNRT & CSNRT & TSACT & SCL \\
\hline Normal $^{\mathrm{a}}$ & $358 \pm 67.32$ & $100 \pm 27.33$ & $58.12 \pm 9.71$ & $246 \pm 23.06$ \\
SND $^{\mathrm{a}}$ & $590 \pm 89.12$ & $190 \pm 49.63$ & $93.2 \pm 12.9$ & $398 \pm 52.63$ \\
Q-value & 6.568 & 5.022 & 6.86 & 8.315 \\
P-value & $<0.01$ & $<0.01$ & $<0.01$ & $<0.01$
\end{tabular}

SNRT, sinus node recovery time; CSNRT, corrected sinus node recovery time; TSACT, total sinoatrial conduction time; SCL, sinus cycle length. ${ }^{2} 20$ rabbits in total.

Table II. Influence of racemic higenamine on the electrophysiology of normal sinus nodes (mean \pm SD).

\begin{tabular}{lcccc}
\hline Groups & SNRT & CSNRT & TSACT & SCL \\
\hline Treatment $^{\mathrm{a}}$ & $329.6 \pm 10.99$ & $98.2 \pm 12.11$ & $61.1 \pm 3.81$ & $231.4 \pm 15.03$ \\
Control $^{\mathrm{a}}$ & $362.2 \pm 24.58$ & $112.4 \pm 15.33$ & $70.9 \pm 3.79$ & $251.1 \pm 1.34$ \\
Q-value $_{\text {P-value }}^{3.835}$ & $<0.01$ & $<0.05$ & 2.64 & 2.38 \\
\end{tabular}

SNRT, sinus node recovery time; CSNRT, corrected sinus node recovery time; TSACT, total sinoatrial conduction time; SCL, sinus cycle length. ${ }^{\mathrm{a}} 10$ rabbits in total.

of the superior vena cava and right atrium for 5-10 min. An animal electrocardiogram then demonstrated sinus bradycardia, P-wave disappearance, cross-nodal rhythm and sinus arrest or tachycardia-bradycardia syndrome in succession. External application of the formaldehyde-treated dressing was stopped when tachycardia-bradycardia was observed. After stabilizing for $20 \mathrm{~min}$, various electrophysiological indicators of sinoatrial function were determined. The sham operation model of normal sinoatrial function was established with physiological saline instead of formaldehyde solution. This study was carried out in accordance with the recommendations of the Guide for the Care and Use of Laboratory Animals of the National Institutes of Health. The animal use protocol was reviewed and approved by the Institutional Animal Care and Use Committee (IACUC) of Yantaishan Hospital.

Establishment of rabbit heart model and atrial pacing algorithm. One rabbit was anesthetized with $25 \%$ urethane. A thoracotomy in the longitudinal direction was performed through the midline of the sternum and the pericardium was opened along the mediastinum to expose the right auricle as far as possible. The pacing electrode (homemade monopolar electrode) was inserted in the border zone of the root of the superior vena cava and right auricle, and another electrode was inserted into the exposed chest wall muscle to form a circuit. The other end of the electrode was connected to a DF-4A cardiac electrophysiological stimulation meter to produce cardiac pacing and determine various electrophysiological indicators of the sinus node.

Electrophysiological determination of cardiac sinoatrial function. Ten sinus cardiac cycles were recorded and the mean was taken as the SCL. The $\mathrm{S}_{1} \mathrm{~S}_{1}$ grading incremental pacing method was used to determine the SNRT (6). The CSNRT was calculated using the following formula: CSNRT $=$ SNRT - basic sinus cardiac cycle. The continuous pacing method of Narula was used to measure the TSACT (7). The formula used was: TSACT $=$ SP - PP (SP, the period from the latest pacing pulse-S to the first P-wave; PP, sinus cycle length before atrial pacing).

Statistical analysis. Data were expressed as means \pm standard deviation (SD). The means among groups were compared using one-way analysis of variance. The means of two samples were compared using a $\mathrm{Q}$ test. The statistics were analyzed with new drug statistical software (NDST; New Drug Statistical Treatment, Tsinghua University Publishing House, Beijing, China). The $\chi^{2}$ test was used to analyze count data.

\section{Results}

Establishment of models. The 40 rabbits had normal electrocardiograms prior to establishment of models. Following formaldehyde or physiological saline wet dressing of the sinus node area and stabilization for $20 \mathrm{~min}$, the electrophysiological changes of the sinus node were determined, including SCL, SNRT, CSNRT and SACT (Table I).

It was observed that the sinoatrial function was significantly impaired following formaldehyde wet dressing, and SCL, SNRT, CSNRT and SACT were longer than those in the normal group (physiological saline-treated group; $\mathrm{P}<0.01$ ), which indicated that the sinus nodes of the rabbits suffered dysfunction.

Electrophysiology of normal sinus node. The 20 normal rabbits were randomly divided into two groups, the treatment 
Table III. Influence of racemic higenamine on the electrophysiological parameters of SND rabbit sinus node function (mean \pm SD).

\begin{tabular}{lcccr}
\hline Groups & SNRT & CSNRT & TSACT & SCL \\
\hline Treatment $^{\mathrm{a}}$ & $511.5 \pm 56.5$ & $147.3 \pm 34.5$ & $81.4 \pm 16.5$ & $364.2 \pm 29.56$ \\
Control $^{\mathrm{a}}$ & $600.0 \pm 76.1$ & $190.7 \pm 45.5$ & $99.8 \pm 16.2$ & $409.3 \pm 45.53$ \\
Q-value $^{\text {P-value }}$ & 2.952 & 2.403 & 2.511 & 2.78 \\
& $<0.01$ & $<0.05$ & $<0.05$ & $<0.05$ \\
\hline
\end{tabular}

SND, damaged sinus node; SNRT, sinus node recovery time; CSNRT, corrected sinus node recovery time; TSACT, total sinoatrial conduction time; SCL, sinus cycle length. ${ }^{\mathrm{a}} 10$ rabbits in total.

Table IV. Comparison of the influence of racemic higenamine on the electrophysiological parameters of normal sinus node and SND group (mean \pm SD).

\begin{tabular}{lcccc}
\hline Groups & $\Delta$ SNRT & $\Delta$ CSNRT & $\Delta$ TSACT & $\Delta$ SCL \\
\hline Control $^{\mathrm{a}}$ & $32.6 \pm 25.7$ & $14.22 \pm 14.0$ & $9.97 \pm 2.06$ & $19.48 \pm 15.7$ \\
SND $^{\mathrm{a}}$ & $88.5 \pm 31.3$ & $43.4 \pm 24.4$ & $18.4 \pm 8.41$ & $45.1 \pm 16.1$ \\
Q-value $^{\text {P-value }}$ & 4.35 & 3.028 & 5.27 & 3.594 \\
\hline
\end{tabular}

SND, damaged sinus node; SNRT, sinus node recovery time; CSNRT, corrected sinus node recovery time; TSACT, total sinoatrial conduction time; SCL, sinus cycle length. ${ }^{\mathrm{a}} 10$ rabbits in total.

Table V. Therapeutic effect of racemic higenamine on arrhythmia in SND rabbits.

\begin{tabular}{lccc}
\hline Groups & Cured & Not cured & Total \\
\hline Control & 1 & 9 & 10 \\
Treatment & 9 & 1 & 10 \\
Total & 10 & 10 & 20
\end{tabular}

SND, damaged sinus node.

and control groups, which were medicated with $0.04 \mathrm{mg} / \mathrm{kg}$ racemic higenamine and the control groups were administered $10 \mathrm{ml}$ physiological saline via the marginal ear vein within $5 \mathrm{~min}$. After $20 \mathrm{~min}$, various electrophysiological indicators of sinus node function were observed to differ between the treatment and control groups (Table II).

The results demonstrate that the SNRT, CSNRT, SACT and SCL in the treatment group were significantly shorter than those in the control group, which indicated that racemic higenamine increased the normal sinus node self-discipline and accelerated the sinoatrial conduction time.

Electrophysiology of SND rabbits. Twenty SND rabbits were randomly divided into two groups, the treatment and the control groups, after the establishment of models. The rabbits were medicated with $0.04 \mathrm{mg} / \mathrm{kg}$ racemic higenamine and the control groups were administered $10 \mathrm{ml}$ physiological saline via the marginal ear vein within $5 \mathrm{~min}$. After $20 \mathrm{~min}$, various electrophysiological indicators of the sinus node which were compared between the treatment and the control group were observed to differ (Table III).

The results showed that SNRT, CSNRT, SACT and SCL in the treatment group were significantly shorter than those in the control group, which indicated that racemic higenamine was also able to increase the self-discipline and the sinoatrial conduction time of the damaged sinus node.

Effects of racemic higenamine on the electrophysiology of the sinus node. Following medication of the treatment and control groups in the normal sinus node group (sham operation group), the differences in the experimental results of SNRT, CSNRT, SACT and SCL were determined, and the means and SDs were calculated. These were compared with the corresponding experimental results of the treatment and control groups in the SND group to inspect whether the affects of racemic higenamine on different sinoatrial functions of the sinus node were the same in the SND and normal sinus node groups (Table IV).

It was observed that following racemic higenamine treatment, the differences in the electrophysiological parameters between the higenamine-treated and control subgroups of the SND group were significantly larger than those of the normal sinus node group, which indicated that the affect of racemic higenamine on damaged sinus nodes was significantly greater than on normal sinus nodes.

SND rabbit cardiac rhythm. Twenty rabbits had normal electrocardiograms prior to the establishment of models, but a variety of arrhythmia may have been present during the process of establishment. After stabilization for $20 \mathrm{~min}$, the arrhythmia 
revealed by electrocardiograms were used as criteria to identify the therapeutic effect of higenamine on arrhythmia by comparison of the arrhythmia between treatment and control groups (using the $\chi^{2}$ test; Table V). According to the $\chi^{2}$ test, there was a statistical significance when the number of cured cases was in the range of 7-10.

\section{Discussion}

With the continuous improvement of the standard of animal experiments, the animal model of SND with bradyarrhythmia may be established to provide an effective and scientific evaluation tool for SSS diagnosis and treatment. The acute SND model is established by formaldehyde wet dressing of the sinus node area $(8,9)$ and is widely used at present. The establishment of a successful model is mainly determined by the statistical difference between treatment and control groups. Any of the following may be used as indications of a successful individual animal model (10): i) sinus rhythm slowed down by $\sim 50 \%$; ii) atrioventricular junctional escape rhythm; iii) sinus arrest; and iv) fast-slow syndrome. SCL, SNRT, CSNRT and TSACT were significantly prolonged in the SND group compared with the normal sinus node group (all $\mathrm{P}<0.01$ ), which showed that formaldehyde wet dressing of the sinus node area was able to cause SND and the decrease of sinus node self-discipline. The SND model was consistent with clinical SSS in electrophysiological response, stablity and reliability.

In the present study, an in vivo sinoatrial node damage model was established in live animals which were given direct sinoatrial electrode pacing in order to investigate the pathogenesis of SSS, and observe the electrophysiological influence of racemic higenamine on normal and damaged sinoatrial function in rabbits. The present results showed that the SCL, SNRT, CSNRT and TSACT in the racemic higenamine treatment group were significantly shortened compared with those in the control group, which indicated that racemic higenamine clearly shortened the SCL of the sinus node and reduced SNRT and TSACT. These observations are similar to the isoproterenol-like effects of B-receptor stimulants and may be associated with racemic higenamine promotion of the influx of $\mathrm{Ca}^{2+}$ and $\mathrm{Na}^{+}$in the action potential plateau. The influx of $\mathrm{Ca}^{2+}$ may promote repolarizing $\mathrm{K}^{+}$currents $(11,12)$ to accelerate the speed of action potential repolarization and shorten action potential duration, which may accelerate the sinus node cells' self-discipline and sinoatrial and atrioventricular conduction. Kimura et al (13) investigated whether higenamine had positive chronotropic and inotropic effects on isolated rat atria. Higenamine had a cardiotonic effect on isolated heart by enhancing the myocardial contractility, increasing contraction amplitude and accelerating the sinus rhythm, and the effects on cardiac rhythm and heart rate were closely associated with the dose (14). Yu et al (12) observed that aconite I was clearly able to heighten Purkinje fiber self-discipline and shorten the action potential duration when perfused into the pseudotendon and right ventricular papillary muscle of dogs in vitro, but there were no significant changes to the effective refractory period. However, isoprenaline shortened the Purkinje fiber action potential duration and shortened the effective refractory period of Purkinje fiber action potential, which indicated that the mechanisms were not the same. In the clinic, isoproter- enol and aconite I are both used for the treatment of various types of bradycardia and atrioventricular block. However, isoproterenol clearly shortens the effective refractory period of Purkinje fiber action potentia and, therefore, has adverse reactions such as serious arrhythmia, which may even be very serious in some cases. By contrast, aconite I is able to extend the effective refractory period of Purkinje fibers, action potential duration/effective refractory period (APD/ERP), but not shorten the effective refractory period, so that the probability of ventricular arrhythmia in clinical application is greatly decreased, indicating that aconite I may be superior to isoproterenol in the treatment of arrhythmia. This is also supported by the present study in which no ventricular arrhythmia was observed during the experimental process.

The experimental results of the SND and normal sinus node groups showed that differences in various physiological parameters of the sinus node in the SND group, including $\triangle \mathrm{SNRT}, \triangle \mathrm{CSNRT}, \triangle \mathrm{SACT}$ and $\triangle \mathrm{SCL}$, were markedly larger than those in the normal sinus node group, with a significant statistical significance (all $\mathrm{P}<0.01$ ). This indicated that racemic higenamine had a more evident effect on damaged sinus nodes than on normal sinus nodes and had a protective effect on SND. A possible mechanism is that racemic higenamine has a $\beta$-adrenergic excitatory-like effect. The mechanism may also relate to the following: i) racemic higenamine may increase coronary flow and improve the blood supply of the sinus node to improve the sinoatrial function and significantly inhibits myocardial ischemia and arrhythmia $(15,16)$. Animal experiments have demonstrated that racemic higenamine is able to inhibit platelet aggregation, with an antithrombotic function, and improve disseminated intravascular coagulation $(17,18)$ and myocardial ischemia-reperfusion myocardial damage. Racemic higenamine has been reported to have a marked protective effect on hypoxic-ischemic animals (19), and may reduce and relieve the range and extent of acute myocardial damage. ii) The mechanism may involve an anti-inflammatory effect. Experiments in animals have shown that racemic higenamine restrains inflammatory reaction $(20,21)$, increases diastolic bronchial wall, increases oxygen supply and protects the cardiopulmonary function. After formaldehyde wet dressing, inflammatory exudation and protein denaturation appeared in the sinus node area, causing different degrees of damage to the tissues, blood circulation and pacemaker cell selfdiscipline in the sinus nodes and leading to sinus heart rate deceleration, or even arrest, ST segment shift and other ischemic changes. Following medication, racemic higenamine inhibited vascular inflammatory exudation and reduced the inflammatory changes of tissues surrounding the sinus node, which significantly inhibited the swelling of sinus node tissues. Racemic higenamine may also improve the blood supply in the sinus node area, reduce myocardial ischemia range and inhibits arrhythmia.

The experimental results of the present study confirm that racemic higenamine increases sinus node self-discipline, improves sinoatrial and atrioventricular conduction, and has a marked therapeutic effect on SSS, with few side effects. Therefore, it is of value as a clinical treatment. The study is partly limited since it is based on an acutely damaged sinus node. 


\section{References}

1. Da Costa A, Kirkorian G, Cucherat M, et al: Antibiotic prophylaxis for permanent pacemaker implantation: a meta-analysis. Circulation 97: 1796-1801, 1998.

2. Bai G, Yang Y, Shi Q, Liu Z, Zhang Q and Zhu YY: Identification of higenamine in Radix Aconiti Lateralis Preparata as a beta2-adrenergic receptor agonist1. Acta Pharmacol Sin 29: 1187-1194, 2008

3. Pyo MK, Lee DH, Kim DH, et al: Enantioselective synthesis of $(\mathrm{R})-(+)-$ and $(\mathrm{S})-(-)$-higenamine and their analogues with effects on platelet aggregation and experimental animal model of disseminated intravascular coagulation. Bioorg Med Chem Lett 18: 4110-4114, 2008.

4. Pyo MK, Kim JM, Jin JL, Chang KC, Lee DH and Yun-Choi HS Effects of higenamine and its 1-naphthyl analogs, YS-49 and YS-51, on platelet TXA2 synthesis and aggregation. Thromb Res 120: 81-86, 2007.

5. Praman S, Mulvany MJ, Williams DE, Andersen RJ and Jansakul C: Hypotensive and cardio-chronotropic constituents of Tinospora crispa and mechanisms of action on the cardiovascular system in anesthetized rats. J Ethnopharmacol 140 166-178, 2012.

6. Narula OS, Samet P and Javier RP: Significance of the sinus-node recovery time. Circulation 45: 140-158, 1972.

7. Narula OS, Shantha N, Vasquez M, Towne WD and Linhart JW: A new method for measurement of sinoatrial conduction time. Circulation 58: 706-714, 1978.

8. Sealy WC, Bache RJ, Seaber AV and Bhattacharga SK: The atrial pacemaking site after surgical exclusion of the sinoatrial node. J Thorac Cardiovasc Surg 65: 841-850, 1973.

9. Zhou SH, Song ZY, Yao Q and Li YH: Dynamic state of electrophysiology function of sinoatrial node after its constitution damaged acutely. Chin J Cardiac Pacing Electrophysiol 18 : 127-129, 2004 (In Chinese).

10. Li YK: Methodology of Pharmacological Experiment for the Traditional Chinese Medicine. Shanghai Scientific and Technology Press, Shanghai, p283, 1991 (In Chinese).

11. Liu W, Sato Y, Hosoda Y, Hirasawa K and Hanai H: Effects of higenamine on regulation of ion transport in guinea pig distal colon. Jpn J Pharmacol 84: 244-251, 2000.
12. Yu GR, Qiao SY, Zheng DS, Huang MX and Bian YT: Effect of higenamine on action potential of Purkinje's cells and ventricular myocardial cells in dogs. J Tradit Chin Med 4: 133-140, 1984

13. Kimura I, Makino M, Takamura Y, Islam MA and Kimura M: Positive chronotropic and inotropic effects of higenamine and its enhancing action on the aconitine-induced tachyarrhythmia in isolated murine atria. Jpn J Pharmacol 66: 75-80, 1994.

14. Chong WS, Lee YS, Kang YJ, et al: Comparison of inodilator effect of higenamine, YS49, YS51, tetrahydroisoquinoline analogs, and dobutamine in the rat. Korean J Physiol Pharmacol 2: 323-330, 1998.

15. Wong KK, Lo CF and Chen CM: Endothelium-dependent higenamine-induced aortic relaxation in isolated rat aorta. Planta Med 63: 130-132, 1997.

16. Lee YS, Kang YJ, Kim HJ, et al: Higenamine reduces apoptotic cell death by induction of heme oxygenase-1 in rat myocardial ischemia-reperfusion injury. Apoptosis 11: 1091-1100, 2006.

17. Yun-Choi HS, Pyo MK, Park KM, Chang KC and Lee DH: Anti-thrombotic effects of higenamine. Planta Med 67: 619-622, 2001.

18. Yun-Choi HS, Pyo MK, Chang KC and Lee DH: The effects of higenamine on LPS-induced experimental disseminated intravascular coagulation (DIC) in rats. Planta Med 68: 326-329, 2002.

19. Ha YM, Kim MY, Park MK, et al: Higenamine reduces HMGB1 during hypoxia-induced brain injury by induction of heme oxygenase-1 through PI3K/Akt/Nrf-2 signal pathways. Apoptosis 17: 463-474, 2012

20. Ueki T, Akaishi T, Okumura $\mathrm{H}$ and Abe K. Extract from Nandina domestica inhibits lipopolysaccharide-induced cyclooxygenase-2 expression in human pulmonary epithelial A549 cells. Biol Pharm Bull 35: 1041-1047, 2012.

21. Park JE, Kang YJ, Park MK, et al: Enantiomers of higenamine inhibit LPS-induced iNOS in a macrophage cell line and improve the survival of mice with experimental endotoxemia. Int Immunopharmacol 6: 226-233, 2006. 